\title{
Formulation and analytic calculation for the spin angular momentum of a moonlet due to inelastic collisions of ring particles
}

\author{
Keiji Ohtsuki \\ Laboratory for Atmospheric and Space Physics, University of Colorado, 392 UCB, Boulder, Colorado 80309-0392, U.S.A.
}

(Received February 6, 2004; Revised July 16, 2004; Accepted July 28, 2004)

\begin{abstract}
Small moonlets embedded in planetary rings acquire spin angular momentum by inelastic collisions of a number of ring particles. We obtain analytic expressions for the mean and mean square spin angular momenta delivered to a moonlet in the high-velocity case where mutual gravity between the moonlet and particles can be neglected. We find that the mean angular momentum brought by a large number of small impacts would result in an equilibrium rotation of a moonlet in the prograde direction that is slower than the synchronous rotation, while large impacts would significantly affect the rotation when the mass of largest impactors is comparable to the moonlet's mass and/or the velocity dispersion of particles is larger than the Kepler shear across the moonlet's radius. We present a new formulation that allows a unified analysis of these two components of moonlet rotation, and confirmed the validity of this formulation and the above analytic calculations using N-body simulation.
\end{abstract}

Key words: Celestial mechanics, rotational dynamics, planetary rings, origin of solar system.

\section{Introduction}

A number of small satellites have been found in Saturn's rings, and it is believed that there are a large number of unseen moonlets embedded in the rings. Isolated large moons ( $\gtrsim 100 \mathrm{~m}$ ) would be tidally despun to rotate synchronously, but smaller moonlets embedded in rings must be spun up by collisions with other particles (Weidenschilling et al., 1984). When particles' velocity dispersion is much larger than the escape velocity of a moonlet, mutual gravity between particles and the moonlet can be neglected, and we can evaluate analytically the angular momentum delivered to the moonlet by collisions of particles. In previous studies on planetary rotation, mean and mean square angular momenta that a planet acquires by accretion of planetesimals have been evaluated both analytically and numerically (e.g., Giuli, 1968; Tanikawa et al., 1989; Ida and Nakazawa, 1990; Lissauer and Kary, 1991; Lissauer and Safronov, 1991; Dones and Tremaine, 1993a, b; Lissauer et al., 1997; Ohtsuki and Ida, 1998; see a review by Lissauer et al., 2000). In these studies, it is assumed that planetesimals are accreted by the planet whenever they collide. However, in ring-satellite systems where the tidal force of the central planet is important, inelastic rebound of particles needs to be taken into account.

On the other hand, it has been shown that two components need to be taken into account in the study of planetary rotation: One is the systematic component of rotation that a planet obtains from a disk of small planetesimals, and the other is the random component imparted by large impactors. Dones and Tremaine (1993a, b) derived a formulation for planetary rotation due to planetesimal accumulation that allows a unified treatment of the two components. Previ-

Copy right (C) The Society of Geomagnetism and Earth, Planetary and Space Sciences (SGEPSS); The Seismological Society of Japan; The Volcanological Society of Japan; The Geodetic Society of Japan; The Japanese Society for Planetary Sciences; TERRA PUB ous studies related to rotation of ring particles often assume that they have identical sizes (e.g., Araki, 1988, 1991). Salo (1987a, b) studied rotation of ring particles using analytic calculation and numerical simulation. In the analytic calculation, Kepler motion of particles was taken into account in an approximate manner, by assuming an isotropic distribution of random velocities. He primarily studied the case of identical particles: the case of a power-law size distribution was also examined, but the relative contribution of the systematic and the random components was not discussed. Recently, Morishima and Salo (2004) obtained the systematic component of moonlet rotation by three-body orbital integration, but they did not evaluate the random component. In order to obtain the two components for moonlet rotation, we need to modify the previous formulation of Dones and Tremaine (1993a, b), which was derived for planetary rotation under the assumption of perfect accretion.

In the present paper, we carry out analytic calculation for the spin angular momentum delivered to a moonlet by collisions of a number of particles, neglecting their mutual gravity. Section 2 describes basic formulation for the analytic calculation, and we present the calculation for the mean and mean square angular momenta in Section 3. In Section 4, we derive a new formulation that allows a unified analysis of the systematic and the random components of moonlet rotation due to inelastic rebound of particles, and we use the results of Section 3 to examine the relative importance of the two components. We find that the contribution of the random component is significant when the mass of largest impactors is comparable to the moonlet's mass and/or the velocity dispersion of particles is larger than the Kepler shear across the moonlet's radius. We confirm the validity of the new formulation and these analytic results using $\mathrm{N}$-body simulation in Section 5. Our results are summarized in Section 6. 
2. Basic Formulation for the Calculation of Spin Angular Momentum

We assume that a moonlet (subscript 1) and a particle (subscript 2) are spheres with the same density, and that their masses, radii, and spin angular velocity vectors are given by $m_{i}, R_{i}$, and $\omega_{i}(i=1,2)$, respectively. We also assume that the moonlet is on a circular orbit with a semimajor axis $a$ and the Keplerian angular velocity $\Omega$. We use Hill's coordinate system centered on the moonlet (e.g., Nakazawa et al., 1989), where the $x$-axis points radially outward, the $y$ axis points in the direction of the moonlet's orbital motion, and the $z$-axis is normal to the $x-y$ plane. The masses of the moonlet and a particle are assumed to be much smaller than the mass of the central planet $M_{\mathrm{c}}$, and eccentricities and inclinations of particles are assumed to be much smaller than unity. Then, equations for the motion of a particle relative to the moonlet can be linearized, and they can be written in nondimensional forms if time is scaled by $\Omega^{-1}$ and length by the Hill radius $R_{\mathrm{H}}=a h$, where $h \equiv\left\{\left(m_{1}+m_{2}\right) / 3 M_{\mathrm{c}}\right\}^{1 / 3}$. Furthermore, the motions of the moonlet and a particle can be separated into the relative motion and the center of mass motion, and the nondimensional equations for the relative motion are written as

$$
\begin{aligned}
& \ddot{\tilde{x}}=2 \dot{\tilde{y}}+3 \tilde{x}-3 \tilde{x} / \tilde{r}^{3}, \\
& \ddot{\tilde{y}}=-2 \dot{\tilde{x}}-3 \tilde{y} / \tilde{r}^{3}, \\
& \ddot{\tilde{z}}=-\tilde{z}-3 \tilde{z} / \tilde{r}^{3},
\end{aligned}
$$

where tildes are used to denote scaled quantities, and $\tilde{r} \equiv$ $\left(\tilde{x}^{2}+\tilde{y}^{2}+\tilde{z}^{2}\right)^{1 / 2}$.

In this paper, we consider the case where mutual gravity between the moonlet and particles can be neglected. Ohtsuki $(1992,1999)$ demonstrated that analytic expressions of collision and velocity stirring rates derived for non-gravitating ring particles agree well with results of three-body orbital integration that takes into account mutual gravity when particles' random velocity is larger than a few times their escape velocity. Such a high velocity would be realized in rings perturbed by other larger satellites. We also confirmed that the results on moonlet rotation presented in this paper agree with three-body orbital integration in such a high-velocity case (Ohtsuki, 2004), although the effect of mutual gravity enhances the rate of angular momentum transfer during particle collisions in lower velocity cases (Morishima and Salo, 2004; Ohtsuki, 2004). However, analytic studies for the above high-velocity limit are useful to understand the numerical results of orbital integration for the gravitating case. They would be also applied to asteroid rotation, where the effect of mutual gravity is less important.

In the non-gravitating case, the last terms in the r.h.s. of Eq. (1) (i.e., those proportional to $-3 / \tilde{r}^{3}$ ) can be omitted, and the relative motion of particles can be written as

$$
\begin{aligned}
& \tilde{x}=\tilde{b}-\tilde{e} \cos (\tilde{t}-\tau), \\
& \tilde{y}=-\frac{3}{2} \tilde{b}(\tilde{t}-\phi)+2 \tilde{e} \sin (\tilde{t}-\tau), \\
& \tilde{z}=\tilde{i} \sin (\tilde{t}-\varpi),
\end{aligned}
$$

where $\tilde{e}$ and $\tilde{i}$ are the eccentricity and the inclination for the relative motion scaled by $h ; \tilde{b}$ is the difference in the semimajor axes of the moonlet and the particle scaled by $R_{\mathrm{H}}$; $\phi$ defines the origin of time; and $\tau$ and $\varpi$ are the horizontal and vertical phase angles, respectively.

First, we describe the formulation and the analytic results for the collision rate of ring particles onto a moonlet based on previous works on planetary accretion rates (Nakazawa et al., 1989; Greenzweig and Lissauer, 1990, 1992), which we will use below. The nondimensional collision rate of particles onto a moonlet can be defined as (Nakazawa et al., 1989)

$$
P_{\mathrm{col}}(\tilde{e}, \tilde{i}) \equiv \int p_{\mathrm{col}}(\tilde{e}, \tilde{i}, \tilde{b}, \tau, \varpi) \frac{3}{2}|\tilde{b}| d \tilde{b} \frac{d \tau d \varpi}{(2 \pi)^{2}},
$$

where $p_{\mathrm{col}}$ is the collision probability between the moonlet and particles with given $\tilde{e}, \tilde{i}$, and $\tilde{b}$, and $p_{\text {col }}=1$ for collision orbits and 0 otherwise. In the high-velocity case where mutual gravity can be neglected, $p_{\text {col }}$ can be evaluated analytically as (Nakazawa et al., 1989)

$$
\begin{aligned}
p_{\mathrm{col}}(\tilde{e}, \tilde{i}, \tilde{b}) & =\int p_{\mathrm{col}}(\tilde{e}, \tilde{i}, \tilde{b}, \tau, \varpi) \frac{d \tau d \varpi}{(2 \pi)^{2}} \\
& =\frac{2 \tilde{r}_{\mathrm{p}}^{2}}{3 \pi \tilde{b} \tilde{i}} \sqrt{\frac{\tilde{e}^{2}+\tilde{i}^{2}-\frac{3}{4} \tilde{b}^{2}}{\tilde{e}^{2}-\tilde{b}^{2}}},
\end{aligned}
$$

where $\tilde{r}_{\mathrm{p}} \equiv\left(R_{1}+R_{2}\right) / R_{\mathrm{H}}$. Then, performing the integral over $\tilde{b}, P_{\text {col }}$ can be obtained as (Nakazawa et al., 1989; Greenzweig and Lissauer, 1990)

$$
\begin{aligned}
P_{\mathrm{col}} & =\int p_{\mathrm{col}}(\tilde{e}, \tilde{i}, \tilde{b}) \frac{3}{2}|\tilde{b}| d \tilde{b} \\
& =\frac{2 \tilde{r}_{\mathrm{p}}^{2} E(k)}{\pi} \sqrt{1+(\tilde{e} / \tilde{i})^{2}},
\end{aligned}
$$

where $E(k)$ is the complete elliptic integral of the second kind with $k=\left[3 \tilde{e}^{2} / 4\left(\tilde{e}^{2}+\tilde{i}^{2}\right)\right]^{1 / 2}$.

On the other hand, N-body simulations show that eccentricities and inclinations of particles in optically thin rings follow the Rayleigh distribution (e.g., Ohtsuki and Emori, 2000) given as

$$
f(\tilde{e}, \tilde{i}) d \tilde{e} d \tilde{i}=\frac{4 \tilde{e} \tilde{i}}{\left\langle\tilde{e}^{2}\right\rangle\left\langle\tilde{i}^{2}\right\rangle} \exp \left(-\frac{\tilde{e}^{2}}{\left\langle\tilde{e}^{2}\right\rangle}-\frac{\tilde{i}^{2}}{\left\langle\tilde{i}^{2}\right\rangle}\right) d \tilde{e} d \tilde{i} .
$$

The Rayleigh distribution average of $P_{\text {col }}$ can be calculated as

$$
\left\langle P_{\mathrm{col}}\right\rangle=\int P_{\mathrm{col}}(\tilde{e}, \tilde{i}) f(\tilde{e}, \tilde{i}) d \tilde{e} d \tilde{i} .
$$

In the high-velocity case, $\left\langle P_{\text {col }}\right\rangle$ can be evaluated analytically (Greenzweig and Lissauer, 1992; Dones and Tremaine, 1993b) as

$$
\left\langle P_{\text {col }}\right\rangle=\frac{\tilde{r}_{\mathrm{p}}^{2}}{\pi} I(\beta),
$$

where $\beta \equiv\left\langle\tilde{i}^{2}\right\rangle^{1 / 2} /\left\langle\tilde{e}^{2}\right\rangle^{1 / 2}$ and

$$
I(\beta) \equiv \int_{0}^{1} \frac{4 E\left(\frac{\sqrt{3}}{2} \sqrt{1-\chi^{2}}\right)}{\left\{\beta+\left(\beta^{-1}-\beta\right) \chi^{2}\right\}^{2}} d \chi .
$$




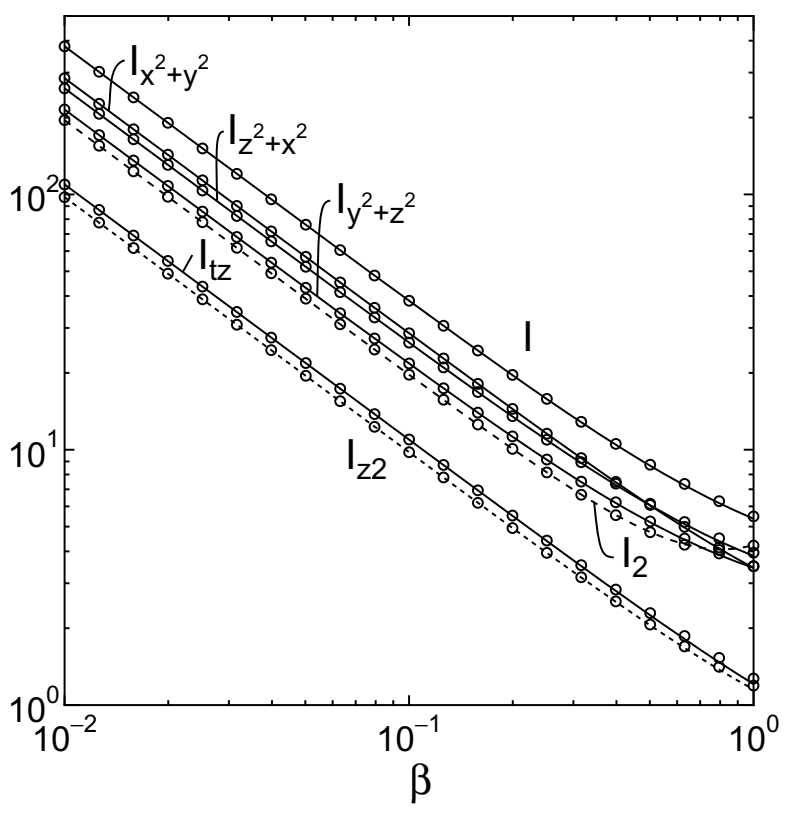

Fig. 1. The plots of the integrals appearing in the analytic expressions The solid lines $\left(I, I_{t z}, I_{x^{2}+y^{2}}, I_{y^{2}+z^{2}}\right.$, and $\left.I_{z^{2}+x^{2}}\right)$ and the dashed lines $\left(I_{2}\right.$ and $I_{z 2}$ ) represent the results obtained by direct numerical integration of these integrals, while the open circles show the results calculated by the approximate analytic expressions described in Appendix (Eqs. (A.7), (A.9), and (A.13))

The plot of $I(\beta)$ is shown in Fig. 1. An approximate analytic expression for $I(\beta)$ is derived by Greenzweig and Lissauer (1992), and is also plotted by the open circles. The method of derivation of the analytic expression is described in Appendix.

The rate of accumulation of spin angular momentum can be defined in a similar manner. Suppose that a moonlet and a particle collide with each other with the relative velocity of the centers of the two bodies in the rotating coordinate system $\boldsymbol{v}$, and that the position of the particle relative to the moonlet at impact is $\boldsymbol{r}$. In general, collisions change the normal and the tangential components of $v$ to the tangent plane. However, as we show below, the change of spin angular momentum of a non-gravitating moonlet depends only on the change of the tangential component of the relative velocity (in the rotating coordinate system), $\boldsymbol{v}_{\mathrm{t}}$. The tangential component of the relative velocity of the two contacting points at impact can be written as (e.g., Araki and Tremaine, 1986)

$$
\boldsymbol{u}_{\mathrm{t}}=\boldsymbol{v}_{\mathrm{t}}+\boldsymbol{\Omega} \times \boldsymbol{r}+\boldsymbol{\lambda} \times\left(R_{1} \omega_{1}+R_{2} \omega_{2}\right),
$$

where $\lambda \equiv \boldsymbol{r} /|\boldsymbol{r}|$ is the unit vector pointing from the center of the moonlet to that of the particle, and $\Omega=(0,0, \Omega)$ is the Keplerian angular velocity vector of the moonlet. In the above, we used the relation $\boldsymbol{r}=\left(R_{1}+R_{2}\right) \lambda$ at impact. We introduce the tangential restitution coefficient $\varepsilon_{\mathrm{t}}$, where $-1 \leq \varepsilon_{\mathrm{t}} \leq 1$ (perfectly smooth spheres have $\varepsilon_{\mathrm{t}}=1$ ). Then, the tangential component of the relative velocity of the two contacting points after impact is given as

$$
\boldsymbol{u}_{\mathrm{t}}^{\prime}=\varepsilon_{\mathrm{t}} \boldsymbol{u}_{\mathrm{t}} .
$$

Using Eq. (11) and conservation of linear and angular mo- menta, the change of $v_{\mathrm{t}}$ can be written as

$$
\begin{aligned}
\Delta \boldsymbol{v}_{\mathrm{t}} & =-\frac{\mathcal{K}}{\mathcal{K}+1} \Delta \boldsymbol{u}_{\mathrm{t}} \\
& =-\frac{\mathcal{K}}{\mathcal{K}+1}\left(1-\varepsilon_{\mathrm{t}}\right)\left\{\boldsymbol{v}_{\mathrm{t}}+R_{\mathrm{p}} \lambda \times(\omega-\boldsymbol{\Omega})\right\},
\end{aligned}
$$

where $\Delta \boldsymbol{u}_{\mathrm{t}} \equiv \boldsymbol{u}_{\mathrm{t}}^{\prime}-\boldsymbol{u}_{\mathrm{t}}, R_{\mathrm{p}} \equiv R_{1}+R_{2}$, and

$$
\boldsymbol{\omega} \equiv\left(R_{1} \omega_{1}+R_{2} \omega_{2}\right) /\left(R_{1}+R_{2}\right) .
$$

$\mathcal{K}$ is the coefficient for the moment of inertia, i.e., $I_{1}=$ $\mathcal{K} m_{1} R_{1}^{2}$, and $\mathcal{K}=2 / 5$ for a homogeneous sphere.

Then, the change of the spin angular momentum of the moonlet can be written as

$$
\begin{aligned}
\Delta \boldsymbol{L}_{1}= & I_{1} \Delta \boldsymbol{\omega}_{1}=R_{1} \lambda \times(-\mu \Delta v) \\
= & \frac{\mathcal{K}}{\mathcal{K}+1} \mu\left(1-\varepsilon_{\mathrm{t}}\right) R_{1} \lambda \\
& \times\left\{v_{\mathrm{t}}+R_{\mathrm{p}} \lambda \times(\omega-\Omega)\right\},
\end{aligned}
$$

where $\mu$ is the reduced mass. If we assume $m_{1} \gg m_{2}$, then $\mu \simeq m_{2}$ and $R_{1} \simeq R_{\mathrm{p}}$. In this case, the specific angular momentum delivered to the moonlet, $\boldsymbol{l}=\Delta \boldsymbol{L} / m_{2}$, can be written as

$$
\begin{gathered}
\boldsymbol{l} \simeq \frac{\mathcal{K}}{\mathcal{K}+1}\left(1-\varepsilon_{\mathrm{t}}\right)\left\{\boldsymbol{l}^{r}+R_{\mathrm{p}}^{2} \lambda \times(\lambda \times(\boldsymbol{\omega}-\boldsymbol{\Omega}))\right\} \\
=\frac{\mathcal{K}}{\mathcal{K}+1}\left(1-\varepsilon_{\mathrm{t}}\right)\left\{\boldsymbol{l}^{r}+R_{\mathrm{p}}^{2}(\boldsymbol{\lambda} \cdot(\boldsymbol{\omega}-\boldsymbol{\Omega})) \boldsymbol{\lambda}\right. \\
\left.-R_{\mathrm{p}}^{2}(\boldsymbol{\omega}-\boldsymbol{\Omega})\right\},
\end{gathered}
$$

where $l^{r}=R_{\mathrm{p}} \lambda \times v_{\mathrm{t}}$ is the translational angular momentum of the particle's motion relative to the moonlet at impact, measured in the rotational coordinate system.

The rate of accumulation of the $z$-component of nondimensional specific angular momentum, $\tilde{l}_{z}=l_{z} / R_{\mathrm{H}}^{2} \Omega$, can be defined as

$$
L_{z} \equiv \int \tilde{l}_{z} p_{\mathrm{col}}(\tilde{e}, \tilde{i}, \tilde{b}, \tau, \varpi) \frac{3}{2}|\tilde{b}| d \tilde{b} \frac{d \tau d \varpi}{(2 \pi)^{2}} .
$$

Using Eqs. (3) and (16), we obtain the $z$-component of the mean angular momentum accreted per unit mass of impacting particles as ${ }^{1}$

$$
\left\langle l_{z}\right\rangle=R_{\mathrm{H}}^{2} \Omega L_{z} / P_{\text {col }} .
$$

Similarly, we define $L_{x}, L_{y},\left\langle l_{x}\right\rangle$, and $\left\langle l_{y}\right\rangle$. Also, we define the rate of accumulation of the square of the total nondimensional angular momentum $\left(\tilde{l}^{2}=l^{2} /\left(R_{\mathrm{H}}^{2} \Omega\right)^{2}\right.$, where $\left.l^{2}=l_{x}^{2}+l_{y}^{2}+l_{z}^{2}\right)$, and that of $\tilde{l}_{z}^{2}=l_{z}^{2} /\left(R_{\mathrm{H}}^{2} \Omega\right)^{2}$, as

$$
\begin{aligned}
& L_{2} \equiv \int \tilde{l}^{2} p_{\mathrm{col}}(\tilde{e}, \tilde{i}, \tilde{b}, \tau, \varpi) \frac{3}{2}|\tilde{b}| d \tilde{b} \frac{d \tau d \varpi}{(2 \pi)^{2}}, \\
& L_{z 2} \equiv \int \tilde{l}_{z}^{2} p_{\mathrm{col}}(\tilde{e}, \tilde{i}, \tilde{b}, \tau, \varpi) \frac{3}{2}|\tilde{b}| d \tilde{b} \frac{d \tau d \varpi}{(2 \pi)^{2}} .
\end{aligned}
$$

\footnotetext{
${ }^{1}$ Note that the brackets in Eqs. (17) and (19) represent the ensemble average over the collision orbits with a given pair of $\tilde{e}$ and $\tilde{i}$. We will use the same expression to denote the Rayleigh distribution averages of these quantities $\left(\left\langle l_{z}\right\rangle,\left\langle l^{2}\right\rangle^{1 / 2}\right.$, and $\left\langle l_{z}^{2}\right\rangle^{1 / 2}$; see Eqs. (34) and (48)), since we think that it is not confusing.
} 
Then, the mean squares $\left\langle l^{2}\right\rangle$ and $\left\langle l_{z}^{2}\right\rangle$ can be given as

$$
\begin{aligned}
& \left\langle l^{2}\right\rangle=\left(R_{\mathrm{H}}^{2} \Omega\right)^{2} L_{2} / P_{\mathrm{col}} \\
& \left\langle l_{z}^{2}\right\rangle=\left(R_{\mathrm{H}}^{2} \Omega\right)^{2} L_{z 2} / P_{\mathrm{col}}
\end{aligned}
$$

The Rayleigh distribution averages such as $\left\langle L_{z}\right\rangle$ and $\left\langle L_{2}\right\rangle$ can be also defined, in a similar manner to the case of $\left\langle P_{\text {col }}\right\rangle$ defined by Eq. (7).

\section{Analytic Calculation of the Mean and Mean Square Angular Momenta}

3.1 Mean angular momentum

Each component of Eq. (15) can be written as

$$
\begin{aligned}
& l_{x}=\frac{\mathcal{K}}{\mathcal{K}+1}\left(1-\varepsilon_{t}\right)\left\{l_{x}^{t}-\left(y^{2}+z^{2}\right) \omega_{x}+x\left(y \omega_{y}+z \omega_{z}\right)\right\}, \\
& l_{y}=\frac{\mathcal{K}}{\mathcal{K}+1}\left(1-\varepsilon_{t}\right)\left\{l_{y}^{t}-\left(z^{2}+x^{2}\right) \omega_{y}+y\left(z \omega_{z}+x \omega_{x}\right)\right\}, \\
& l_{z}=\frac{\mathcal{K}}{\mathcal{K}+1}\left(1-\varepsilon_{t}\right)\left\{l_{z}^{t}-\left(x^{2}+y^{2}\right) \omega_{z}+z\left(x \omega_{x}+y \omega_{y}\right)\right\},
\end{aligned}
$$

where

$$
\begin{aligned}
& l_{x}^{t}=y \dot{z}-z \dot{y}-\Omega x z \\
& l_{y}^{t}=z \dot{x}-x \dot{z}-\Omega y z \\
& l_{z}^{t}=x \dot{y}-y \dot{x}+\Omega\left(x^{2}+y^{2}\right)
\end{aligned}
$$

are the components of the translational angular momentum of the impacting particle in the inertial coordinate system centered on the moonlet (e.g., Lissauer and Kary, 1991).

The mean angular momentum $\left\langle l_{x}\right\rangle,\left\langle l_{y}\right\rangle$, and $\left\langle l_{z}\right\rangle$ in the three-dimensional case with non-zero orbital inclinations can be evaluated analytically when (i) $\tilde{e}, \tilde{i} \gg \tilde{v}_{\mathrm{e}}\left(\tilde{v}_{\mathrm{e}} \equiv v_{\mathrm{e}} / R_{\mathrm{H}} \Omega\right.$ is the scaled escape velocity) and (ii) $\tilde{e}, \tilde{i} \gg \tilde{r}_{\mathrm{p}}$. In this case, the relative velocity at $(\tilde{x}, \tilde{y}, \tilde{z})=(0,0,0)$ in the non-gravitating solution to Eqs. (1) is given by (Nakazawa et al., 1989)

$$
(\dot{\tilde{x}}, \dot{\tilde{y}}, \dot{\tilde{z}})=\left( \pm\left(\tilde{e}^{2}-\tilde{b}^{2}\right)^{1 / 2}, \tilde{b} / 2, \pm \tilde{i}\right)
$$

where each of the four combinations of the signs of $\dot{\tilde{x}}$ and $\dot{\tilde{z}}$ respectively correspond to each of the four bands of collision orbits for given $\tilde{e}, \tilde{i}$, and and $\tilde{b}$. Owing to the symmetry among these four collision bands, the last terms in Eqs. (20) cancel out. Also, $\left\langle l_{x}^{t}\right\rangle=\left\langle l_{y}^{t}\right\rangle=0$, by symmetry with respect to the $z=0$ plane. These characteristics simplify the derivation of analytic expressions of the mean angular momentum.

First, we derive the analytic expression for $L_{z}$ defined by Eq. (16). Here, we define

$$
\begin{aligned}
& L_{t z} \equiv \int \tilde{l}_{z}^{t} p_{\mathrm{col}}(\tilde{e}, \tilde{i}, \tilde{b}, \tau, \varpi) \frac{3}{2}|\tilde{b}| d \tilde{b} \frac{d \tau d \varpi}{(2 \pi)^{2}} \\
& L_{x^{2}+y^{2}} \equiv \int\left(\tilde{x}^{2}+\tilde{y}^{2}\right) p_{\mathrm{col}}(\tilde{e}, \tilde{i}, \tilde{b}, \tau, \varpi) \frac{3}{2}|\tilde{b}| d \tilde{b} \frac{d \tau d \varpi}{(2 \pi)^{2}}
\end{aligned}
$$

where $\tilde{l}_{z}^{t}=l_{z}^{t} /\left(R_{\mathrm{H}}^{2} \Omega\right)$ and $\tilde{x}^{2}+\tilde{y}^{2}=\left(x^{2}+y^{2}\right) / R_{\mathrm{H}}^{2}$. From Eqs. (16) and (20), $L_{z}$ can be written in terms of these quantities as

$$
L_{z}=\frac{\mathcal{K}}{\mathcal{K}+1}\left(1-\varepsilon_{\mathrm{t}}\right)\left(L_{t z}-L_{x^{2}+y^{2}} \cdot \omega_{z} / \Omega\right)
$$

The analytic expression for $L_{t z}$ is obtained by Lissauer $e t a l$. (1997) as

$$
\begin{aligned}
L_{t z}= & \frac{\tilde{r}_{\mathrm{p}}^{4}\left(\tilde{e}^{2}+\tilde{i}^{2}\right)^{1 / 2}}{\pi \tilde{i}} \\
& \times\left(E(k)-\frac{\tilde{e}^{2}+3 \tilde{i}^{2}}{4\left(\tilde{e}^{2}+\tilde{i}^{2}\right)} K(k)+\frac{1}{12 \pi}\right),
\end{aligned}
$$

where $K(k)$ is the complete elliptic integral of the first kind. Note that the last term on the r.h.s. of Eq. (25) accounts for self-shadowing by the moonlet for those orbits with small $\tilde{b}\left(<2 \tilde{r}_{\mathrm{p}} / 3 \pi\right)$ where the motion of the guiding center of a particle during one epicyclic period is less than the moonlet's diameter (Lissauer and Kary, 1991; Dones and Tremaine, 1993b). This correction needs to be taken into account for the evaluation of $L_{t z}$ because of near cancellation of the contributions of the positive and negative angular momenta in the bulk region with $2 \tilde{r}_{\mathrm{p}} / 3 \pi<\tilde{b} \leq \tilde{e}$. On the other hand, such a correction is not necessary for $P_{\text {col }}, L_{x^{2}+y^{2}}, L_{2}$, and $L_{z 2}$, where such cancellation does not occur. In order to obtain the analytic expression for $L_{x^{2}+y^{2}}$, we introduce a new coordinate system $\left(\tilde{x}^{\prime}, \tilde{y}^{\prime}, \tilde{z}^{\prime}\right)$ defined as (Ohtsuki, 1992, 1999)

$$
\begin{aligned}
\left(\begin{array}{c}
\tilde{x} \\
\tilde{y} \\
\tilde{z}
\end{array}\right)= & \left(\begin{array}{ccc}
\sin \theta_{0} & \cos \theta_{0} & 0 \\
-\cos \theta_{0} & \sin \theta_{0} & 0 \\
0 & 0 & 1
\end{array}\right) \\
& \times\left(\begin{array}{ccc}
\sin \theta_{1} & 0 & \cos \theta_{1} \\
0 & 1 & 0 \\
-\cos \theta_{1} & 0 & \sin \theta_{1}
\end{array}\right)\left(\begin{array}{c}
\tilde{x}^{\prime} \\
\tilde{y}^{\prime} \\
\tilde{z}^{\prime}
\end{array}\right)
\end{aligned}
$$

with

$$
\begin{aligned}
& \cos \theta_{0} \equiv \frac{\tilde{b}}{2}\left(\tilde{e}^{2}-\frac{3}{4} \tilde{b}^{2}\right)^{-1 / 2}, \\
& \sin \theta_{0} \equiv\left(\tilde{e}^{2}-\tilde{b}^{2}\right)^{1 / 2}\left(\tilde{e}^{2}-\frac{3}{4} \tilde{b}^{2}\right)^{-1 / 2}, \\
& \cos \theta_{1} \equiv \tilde{i}\left(\tilde{e}^{2}+\tilde{i}^{2}-\frac{3}{4} \tilde{b}^{2}\right)^{-1 / 2}, \\
& \sin \theta_{1} \equiv\left(\tilde{e}^{2}-\frac{3}{4} \tilde{b}^{2}\right)^{1 / 2}\left(\tilde{e}^{2}+\tilde{i}^{2}-\frac{3}{4} \tilde{b}^{2}\right)^{-1 / 2} .
\end{aligned}
$$

Note that the magnitude of each component of the impact velocity under the present approximation is $\left(\left(\tilde{e}^{2}-\tilde{b}^{2}\right)^{1 / 2}, \tilde{b} / 2, \tilde{i}\right)$ (Eq. (22)). The magnitude of the impact velocity is $\left(\tilde{e}^{2}+\tilde{i}^{2}-3 \tilde{b}^{2} / 4\right)^{1 / 2}$, while the magnitude of the impact velocity projected onto the $\tilde{z}=0$ plane is $\left(\tilde{e}^{2}-3 \tilde{b}^{2} / 4\right)^{1 / 2}$. Therefore, $\theta_{0}$ in the above equations represents the direction of an incoming particle in the $\tilde{x}-\tilde{y}$ plane, while $\theta_{1}$ denotes the angle between the direction of the incoming particle and the $\tilde{z}$-axis. Using Eqs. (26) and (27), we 
can express $\tilde{x}^{2}+\tilde{y}^{2}$ in terms of $\left(\tilde{x}^{\prime}, \tilde{y}^{\prime}, \tilde{z}^{\prime}\right)$. Taking averages of $\tilde{x}^{2}+\tilde{y}^{2}$ over $\tilde{y}^{\prime}$ and $\tilde{z}^{\prime}$ (which corresponds to averaging over orbital phases) and integrating over $\tilde{b}$ together with the collision probability $p_{\text {col }}(\tilde{e}, \tilde{i}, \tilde{b})$ given by Eq. (4), we obtain

$$
L_{x^{2}+y^{2}}=\frac{\tilde{r}_{\mathrm{p}}^{4}\left(\tilde{e}^{2}+\tilde{i}^{2}\right)^{1 / 2}}{\pi \tilde{i}}\left(\frac{3}{2} E(k)-\frac{\tilde{i}^{2}}{2\left(\tilde{e}^{2}+\tilde{i}^{2}\right)} K(k)\right) .
$$

From Eqs. (24), (25), and (28), we obtain

$$
\begin{aligned}
L_{z}= & \frac{\mathcal{K}}{\mathcal{K}+1}\left(1-\varepsilon_{\mathrm{t}}\right) \frac{\tilde{r}_{\mathrm{p}}^{4}\left(\tilde{e}^{2}+\tilde{i}^{2}\right)^{1 / 2}}{\pi \tilde{i}} \\
\times & \left\{\left(E(k)-\frac{\tilde{e}^{2}+3 \tilde{i}^{2}}{4\left(\tilde{e}^{2}+\tilde{i}^{2}\right)} K(k)+\frac{1}{12 \pi}\right)\right. \\
& \left.-\left(\frac{3}{2} E(k)-\frac{\tilde{i}^{2}}{2\left(\tilde{e}^{2}+\tilde{i}^{2}\right)} K(k)\right) \frac{\omega_{z}}{\Omega}\right\} .
\end{aligned}
$$

Using Eqs. (5) and (29), we obtain the expression for $\left\langle l_{z}\right\rangle$ for the case where all the particles have identical eccentricities and inclinations, as

$$
\left\langle l_{z}\right\rangle=\frac{\mathcal{K}}{\mathcal{K}+1}\left(1-\varepsilon_{\mathrm{t}}\right)\left(\left\langle l_{z}^{t}\right\rangle-\left\langle x^{2}+y^{2}\right\rangle \omega_{z}\right)
$$

where

$$
\begin{aligned}
& \left\langle l_{z}^{t}\right\rangle=R_{\mathrm{p}}^{2} \Omega\left\{\frac{1}{2}-\frac{\tilde{e}^{2}+3 \tilde{i}^{2}}{8\left(\tilde{e}^{2}+\tilde{i}^{2}\right)} \frac{K(k)}{E(k)}+\frac{1}{24 \pi E(k)}\right\}, \\
& \left\langle x^{2}+y^{2}\right\rangle=R_{\mathrm{p}}^{2}\left\{\frac{3}{4}-\frac{\tilde{i}^{2}}{4\left(\tilde{e}^{2}+\tilde{i}^{2}\right)} \frac{K(k)}{E(k)}\right\} .
\end{aligned}
$$

Equation (30) can be also written in the form $\left\langle l_{z}\right\rangle \propto \omega_{\mathrm{eq}}-\omega_{z}$, where $\omega_{\mathrm{eq}}=\left\langle l_{z}^{t}\right\rangle /\left\langle x^{2}+y^{2}\right\rangle$, which suggests the existence of an equilibrium rotation for which $\left\langle l_{z}\right\rangle$ vanishes. We have $\omega_{\mathrm{eq}}=0.3665 \Omega$ for $e=2 i$.

Following Ohtsuki (1999), who calculated the Rayleigh distribution averages of the viscous stirring and dynamical friction rates of ring particles, we can evaluate the Rayleigh distribution average of $L_{z}$ as

$$
\left\langle L_{z}\right\rangle=\frac{\mathcal{K}}{\mathcal{K}+1}\left(1-\varepsilon_{\mathrm{t}}\right) \frac{\tilde{r}_{\mathrm{p}}^{4}}{\pi}\left\{I_{t z}(\beta)-I_{x^{2}+y^{2}}(\beta)\left(\omega_{z} / \Omega\right)\right\},
$$

where

$$
\begin{aligned}
& I_{t z}(\beta) \equiv \int_{0}^{1} \\
& \frac{2 E\left(\frac{\sqrt{3}}{2} \sqrt{1-\chi^{2}}\right)-\left(\frac{1}{2}+\chi^{2}\right) K\left(\frac{\sqrt{3}}{2} \sqrt{1-\chi^{2}}\right)+\frac{1}{6 \pi}}{\left\{\beta+\left(\beta^{-1}-\beta\right) \chi^{2}\right\}^{2}} d \chi, \\
& I_{x^{2}+y^{2}}(\beta) \\
& \equiv \int_{0}^{1} \frac{3 E\left(\frac{\sqrt{3}}{2} \sqrt{1-\chi^{2}}\right)-\chi^{2} K\left(\frac{\sqrt{3}}{2} \sqrt{1-\chi^{2}}\right)}{\left\{\beta+\left(\beta^{-1}-\beta\right) \chi^{2}\right\}^{2}} d \chi .
\end{aligned}
$$

We show the plots of $I_{t z}(\beta)$ and $I_{x^{2}+y^{2}}(\beta)$ in Fig. 1. We derived approximate analytic expressions for these quantities in Appendix, and are also plotted in Fig. 1 with the open circles. Using Eqs. (8) and (32), we obtain the analytic expression of $\left\langle l_{z}\right\rangle$ in the case with the Rayleigh distribution of particles' eccentricities and inclinations as

$$
\left\langle l_{z}\right\rangle=C_{z}(\beta) \frac{\mathcal{K}}{\mathcal{K}+1}\left(1-\varepsilon_{\mathrm{t}}\right) R_{\mathrm{p}}^{2}\left(\omega_{\mathrm{eq}}-\omega_{z}\right)
$$

where

$$
\begin{aligned}
& C_{z}(\beta) \equiv I_{x^{2}+y^{2}}(\beta) / I(\beta), \\
& \omega_{\mathrm{eq}} \equiv\left\{I_{t z}(\beta) / I_{x^{2}+y^{2}}(\beta)\right\} \Omega .
\end{aligned}
$$

When $\beta=1 / 2$, we have $C_{z}=0.6998$ and $\omega_{\mathrm{eq}}=0.3712 \Omega$. The above value of the equilibrium rotation rate is slightly larger than the results of previous analytic studies on rotating ring particles with identical sizes (Salo, 1987a; Araki, 1991), which gave $\omega_{\mathrm{eq}} \sim 0.3 \Omega$. In the limit of $\beta \ll 1$, all of $I, I_{t z}$, and $I_{x^{2}+y^{2}}$ are proportional to $\beta^{-1}$ (Eqs. (A.8) and (A.14)). As a result, both $C_{z}$ and $\omega_{\mathrm{eq}}$ become independent of $\beta$ in this limit, and we have $C_{z}=0.75$ and $\omega_{\text {eq }}=0.3837 \Omega$. Note that $\beta \simeq 1 / 2$ is expected in such high-velocities that $\left\langle\tilde{e}^{2}\right\rangle^{1 / 2} \gtrsim 2$, where the random velocity dominates particles' relative velocity. On the other hand, $\beta$ can take on smaller values if the velocity stirring of particles occurs in the lower-velocity regime with $\left\langle\tilde{e}^{2}\right\rangle^{1 / 2} \lesssim 1$, where Kepler shear dominates the relative velocity (Ohtsuki, 1999).

Analytic expressions of the $x$ - and the $y$-components of the mean angular momentum can be also derived in a similar manner. We obtain

$$
\begin{aligned}
L_{x}= & -\frac{\mathcal{K}}{\mathcal{K}+1}\left(1-\varepsilon_{\mathrm{t}}\right) \frac{\tilde{r}_{\mathrm{p}}^{4}\left(\tilde{e}^{2}+\tilde{i}^{2}\right)^{1 / 2}}{\pi \tilde{i}} \\
& \times\left\{\frac{5}{6} E(k)+\frac{\tilde{e}^{2}+4 \tilde{i}^{2}}{6\left(\tilde{e}^{2}+\tilde{i}^{2}\right)} K(k)\right\} \frac{\omega_{x}}{\Omega}, \\
L_{y}= & -\frac{\mathcal{K}}{\mathcal{K}+1}\left(1-\varepsilon_{\mathrm{t}}\right) \frac{\tilde{r}_{\mathrm{p}}^{4}\left(\tilde{e}^{2}+\tilde{i}^{2}\right)^{1 / 2}}{\pi \tilde{i}} \\
& \times\left\{\frac{5}{3} E(k)-\frac{1}{6} K(k)\right\} \frac{\omega_{y}}{\Omega},
\end{aligned}
$$


and their Rayleigh distribution averages are given by

$$
\begin{aligned}
& \left\langle L_{x}\right\rangle=-\frac{\mathcal{K}}{\mathcal{K}+1}\left(1-\varepsilon_{\mathrm{t}}\right) \frac{\tilde{r}_{\mathrm{p}}^{4}}{\pi} I_{y^{2}+z^{2}}(\beta) \omega_{x} / \Omega, \\
& \left\langle L_{y}\right\rangle=-\frac{\mathcal{K}}{\mathcal{K}+1}\left(1-\varepsilon_{\mathrm{t}}\right) \frac{\tilde{r}_{\mathrm{p}}^{4}}{\pi} I_{z^{2}+x^{2}}(\beta) \omega_{y} / \Omega,
\end{aligned}
$$

where

$$
\begin{aligned}
& I_{y^{2}+z^{2}}(\beta) \equiv \frac{1}{3} \int_{0}^{1} \\
& \frac{5 E\left(\frac{\sqrt{3}}{2} \sqrt{1-\chi^{2}}\right)+\left(1+3 \chi^{2}\right) K\left(\frac{\sqrt{3}}{2} \sqrt{1-\chi^{2}}\right)}{\left\{\beta+\left(\beta^{-1}-\beta\right) \chi^{2}\right\}^{2}} d \chi,
\end{aligned}
$$

$$
\begin{aligned}
& I_{z^{2}+x^{2}}(\beta) \\
& \equiv \frac{1}{3} \int_{0}^{1} \frac{10 E\left(\frac{\sqrt{3}}{2} \sqrt{1-\chi^{2}}\right)-K\left(\frac{\sqrt{3}}{2} \sqrt{1-\chi^{2}}\right)}{\left\{\beta+\left(\beta^{-1}-\beta\right) \chi^{2}\right\}^{2}} d \chi .
\end{aligned}
$$

We show the plots of $I_{y^{2}+z^{2}}(\beta)$ and $I_{z^{2}+x^{2}}(\beta)$ in Fig. 1 (solid lines), together with their values calculated by the approximate analytic expressions derived in Appendix (open circles).

Using Eqs. (8) and (37), we obtain

$$
\begin{aligned}
& \left\langle l_{x}\right\rangle=-C_{x}(\beta) \frac{\mathcal{K}}{\mathcal{K}+1}\left(1-\varepsilon_{\mathrm{t}}\right) R_{\mathrm{p}}^{2} \omega_{x}, \\
& \left\langle l_{y}\right\rangle=-C_{y}(\beta) \frac{\mathcal{K}}{\mathcal{K}+1}\left(1-\varepsilon_{\mathrm{t}}\right) R_{\mathrm{p}}^{2} \omega_{y},
\end{aligned}
$$

where

$$
\begin{aligned}
& C_{x}(\beta) \equiv I_{y^{2}+z^{2}}(\beta) / I(\beta), \\
& C_{y}(\beta) \equiv I_{z^{2}+x^{2}}(\beta) / I(\beta) .
\end{aligned}
$$

Note that the magnitude of the $x$ - and the $y$-components of the moonlet's rotation rate defined in the above rotating coordinate system changes with time even without particle collisions (Morishima and Salo, 2004; Ohtsuki, 2004), and Eqs. (39) represent the values for the instantaneous directions of the $x$ - and the $y$-axes. When $\beta=1 / 2$, we have $C_{x}=0.6005$ and $C_{y}=0.6997$. In the limit of $\beta \ll 1$, both $C_{x}$ and $C_{y}$ become independent of $\beta$, and we have $C_{x}=0.5654$ and $C_{y}=0.6846$.

Equations (34) and (39) show that the mean angular momentum of a large number of impacts tends to lead to the equilibrium rotation with $\omega_{\mathrm{eq}}=\left(0,0, \omega_{\mathrm{eq}}\right)$.

\subsection{Mean square angular momentum}

The mean square angular momenta $\left\langle l^{2}\right\rangle$ and $\left\langle l_{z}^{2}\right\rangle$ can be evaluated analytically when the above conditions, (i) $\tilde{e}, \tilde{i} \gg$ $\tilde{v}_{\mathrm{e}}$ and (ii) $\tilde{e}, \tilde{i} \gg \tilde{r}_{\mathrm{p}}$, are satisfied. From the result described in the last subsection, we can expect that $\mathcal{O}(|\omega|) \lesssim \Omega$. In this case, the first and the second terms in the braces on the r.h.s. of Eq. (15) are on the order of $R_{\mathrm{p}} e v_{\mathrm{K}}\left(v_{\mathrm{K}}\right.$ is the circular Keplerian velocity of the moonlet) and $R_{\mathrm{p}}^{2} \Omega$, respectively.
Therefore, the second term can be neglected compared to the first term, since $R_{\mathrm{p}} \Omega / e v_{\mathrm{K}} \ll 1$ under the above assumption (ii). Then, the three components of $\boldsymbol{l}$ given as Eq. (15) scaled by $R_{\mathrm{H}}^{2} \Omega$ can be written in terms of the scaled relative position $(\tilde{x}, \tilde{y}, \tilde{z})$ and the scaled relative velocity $(\dot{\tilde{x}}, \dot{\tilde{y}}, \dot{\tilde{z}})$ at impact, as

$$
\begin{aligned}
& \tilde{l}_{x}=l_{x} / R_{\mathrm{H}}^{2} \Omega=\frac{\mathcal{K}}{\mathcal{K}+1}\left(1-\varepsilon_{\mathrm{t}}\right)(\tilde{y} \dot{\tilde{z}}-\tilde{z} \dot{\tilde{y}}), \\
& \tilde{l}_{y}=l_{y} / R_{\mathrm{H}}^{2} \Omega=\frac{\mathcal{K}}{\mathcal{K}+1}\left(1-\varepsilon_{\mathrm{t}}\right)(\tilde{z} \dot{\tilde{x}}-\tilde{x} \dot{\tilde{z}}), \\
& \tilde{l}_{z}=l_{z} / R_{\mathrm{H}}^{2} \Omega=\frac{\mathcal{K}}{\mathcal{K}+1}\left(1-\varepsilon_{\mathrm{t}}\right)(\tilde{x} \dot{\tilde{y}}-\tilde{y} \dot{\tilde{x}}) .
\end{aligned}
$$

Substituting Eqs. (22), (26), and (27) into Eq. (41), we get

$$
\begin{aligned}
& \tilde{l}^{2}=\left(\frac{\mathcal{K}}{\mathcal{K}+1}\right)^{2}\left(1-\varepsilon_{\mathrm{t}}\right)^{2}\left(\tilde{e}^{2}+\tilde{i}^{2}-\frac{3}{4} \tilde{b}^{2}\right)\left(\tilde{y}^{\prime 2}+\tilde{z}^{\prime 2}\right), \\
& \tilde{l}_{z}^{2}=\left(\frac{\mathcal{K}}{\mathcal{K}+1}\right)^{2}\left(1-\varepsilon_{\mathrm{t}}\right)^{2}\left(\tilde{e}^{2}-\frac{3}{4} \tilde{b}^{2}\right) \tilde{y}^{\prime 2} .
\end{aligned}
$$

Taking averages of $\tilde{l}^{2}$ over $\tilde{y}^{\prime}$ and $\tilde{z}^{\prime}$ and integrating over $\tilde{b}$ together with the collision probability $p_{\text {col }}(\tilde{e}, \tilde{i}, \tilde{b})$ given by Eq. (4), we obtain the expression for the nondimensional accretion rate of mean square angular momentum $L_{2}$ defined by Eq. (18) as

$L_{2}=\left(\frac{\mathcal{K}}{\mathcal{K}+1}\right)^{2}\left(1-\varepsilon_{\mathrm{t}}\right)^{2} \frac{\tilde{r}_{\mathrm{p}}^{4}\left(\tilde{e}^{2}+\tilde{i}^{2}\right)^{3 / 2}}{\pi \tilde{i}}\left(E(k)-k^{2} F(k)\right)$,

where $F(k)$ is defined in terms of $E(k)$ and $K(k)$ as

$$
F(k) \equiv \frac{1}{3 k^{2}}\left[\left(2 k^{2}-1\right) E(k)+\left(1-k^{2}\right) K(k)\right] .
$$

Similarly, we obtain

$$
\begin{aligned}
L_{z 2}= & \left(\frac{\mathcal{K}}{\mathcal{K}+1}\right)^{2}\left(1-\varepsilon_{\mathrm{t}}\right)^{2} \\
& \times \frac{\tilde{r}_{\mathrm{p}}^{4} \tilde{e}^{2}\left(\tilde{e}^{2}+\tilde{i}^{2}\right)^{1 / 2}}{2 \pi \tilde{i}}\left(E(k)-\frac{3}{4} F(k)\right) .
\end{aligned}
$$

The Rayleigh distribution averages of these quantities can be evaluated as

$$
\begin{aligned}
& \left\langle L_{2}\right\rangle=\left(\frac{\mathcal{K}}{\mathcal{K}+1}\right)^{2}\left(1-\varepsilon_{\mathrm{t}}\right)^{2} \frac{I_{2}(\beta) \tilde{r}_{\mathrm{p}}^{4}}{\pi}\left\langle\tilde{e}^{2}\right\rangle, \\
& \left\langle L_{z 2}\right\rangle=\left(\frac{\mathcal{K}}{\mathcal{K}+1}\right)^{2}\left(1-\varepsilon_{\mathrm{t}}\right)^{2} \frac{I_{z 2}(\beta) \tilde{r}_{\mathrm{p}}^{4}}{\pi}\left\langle\tilde{e}^{2}\right\rangle,
\end{aligned}
$$


where

$$
\begin{aligned}
& I_{2}(\beta) \equiv \int_{0}^{1} \\
& \frac{4 E\left(\frac{\sqrt{3}}{2} \sqrt{1-\chi^{2}}\right)-3\left(1-\chi^{2}\right) F\left(\frac{\sqrt{3}}{2} \sqrt{1-\chi^{2}}\right)}{\beta^{-1}\left\{\beta+\left(\beta^{-1}-\beta\right) \chi^{2}\right\}^{3}} d \chi \\
& \frac{\left(1-\chi^{2}\right)\left\{2 E\left(\frac{\sqrt{3}}{2} \sqrt{1-\chi^{2}}\right)-\frac{3}{2} F\left(\frac{\sqrt{3}}{2} \sqrt{1-\chi^{2}}\right)\right\}}{\beta^{-1}\left\{\beta+\left(\beta^{-1}-\beta\right) \chi^{2}\right\}^{3}} d \chi .
\end{aligned}
$$

The plots of $I_{2}(\beta)$ and $I_{z 2}(\beta)$ are shown in Fig. 1. We derived approximate analytic expressions for these integrals in Appendix, and are plotted in Fig. 1 with the open circles. From Eqs. (8) and (46), we obtain

$$
\begin{aligned}
\left\langle l^{2}\right\rangle^{1 / 2} & =R_{\mathrm{H}}^{2} \Omega\left(\left\langle L_{2}\right\rangle /\left\langle P_{\text {col }}\right\rangle\right)^{1 / 2} \\
& =C_{2}(\beta) \frac{\mathcal{K}}{\mathcal{K}+1}\left(1-\varepsilon_{\mathrm{t}}\right) R_{\mathrm{p}} \sigma_{x}, \\
\left\langle l_{z}^{2}\right\rangle^{1 / 2} & =R_{\mathrm{H}}^{2} \Omega\left(\left\langle L_{z 2}\right\rangle /\left\langle P_{\text {col }}\right\rangle\right)^{1 / 2} \\
& =C_{z 2}(\beta) \frac{\mathcal{K}}{\mathcal{K}+1}\left(1-\varepsilon_{\mathrm{t}}\right) R_{\mathrm{p}} \sigma_{x},
\end{aligned}
$$

where $\sigma_{x} \equiv\left(\left\langle e^{2}\right\rangle / 2\right)^{1 / 2} a \Omega$ and

$$
\begin{aligned}
& C_{2}(\beta) \equiv\left\{I_{2}(\beta) / I(\beta)\right\}^{1 / 2}, \\
& C_{z 2}(\beta) \equiv\left\{I_{z 2}(\beta) / I(\beta)\right\}^{1 / 2} .
\end{aligned}
$$

When $\beta=1 / 2$, we have $C_{2}=1.048$ and $C_{z 2}=0.6889$. In the limit of $\beta \ll 1$, both $C_{2}$ and $C_{z 2}$ become independent of $\beta$, and we have $C_{2}=1.013$ and $C_{z 2}=0.7165$.

\section{Systematic and Random Components of Moon- let Rotation}

The result for the mean angular momentum obtained in Section 3.1 (Eq. (34)) shows that the moonlet would achieve the equilibrium rotation rate in the prograde direction with $\boldsymbol{\omega}_{\mathrm{eq}}=\left(0,0, \omega_{\mathrm{eq}}\right)$ after experiencing a large number of small impacts, where $\omega_{\mathrm{eq}}$ is given by Eq. (35). However, in addition to the above systematic components of rotation, the random component imparted by large impacts also needs to be taken into account, as we mentioned above. Dones and Tremaine (1993a, b) derived a unified formulation for the two components for planetary rotation under the assumption that the planet accretes planetesimals whenever they collide. In this case, the mass of the planet is equal to the total mass of accreted planetesimals. On the other hand, in the case of moonlet rotation in non-gravitating case that we consider in the present work, particles rebound after impacts, and the mass of the moonlet is kept constant. In the following, we derive an alternative formulation that allows a unified analysis of the systematic and the random components of moonlet rotation. We also discuss the relative importance of these two components using the results obtained in Section 3.
Suppose that the initial values of the spin angular momentum and the spin angular velocity vector of a moonlet (mass $M$, radius $R$, moment of inertia $I=\mathcal{K} M R^{2}$ ) are given by $\boldsymbol{L}_{0}$ and $\omega_{0}\left(=L_{0} / I\right)$, respectively. The spin angular momentum that the moonlet acquires after $N$ impacts of particles can be written as

$$
\boldsymbol{L}=\sum_{i=1}^{N} m_{i} \boldsymbol{l}_{i},
$$

where $\boldsymbol{l}_{\boldsymbol{i}}$ is the specific angular momentum delivered by the $i$-th impactor $m_{i}$. Then the expectation value of $\boldsymbol{L}$ can be written as

$$
\langle\boldsymbol{L}\rangle=N\langle m\rangle\langle\boldsymbol{l}\rangle
$$

where $\langle\boldsymbol{l}\rangle \equiv\left(\left\langle l_{x}\right\rangle,\left\langle l_{y}\right\rangle,\left\langle l_{z}\right\rangle\right)$ are given by Eqs. (34) and (39). The expectation value of the $i$-component $(i=x, y, z)$ of the final rotation rate of the moonlet, $\left\langle\omega_{\mathrm{f}, i}\right\rangle$, can be given by

$$
\left\langle\omega_{\mathrm{f}, i}\right\rangle=\omega_{0, i}+N\langle m\rangle\left\langle l_{i}\right\rangle / I
$$

From Eqs. (34), (39), (51), and (52), we find that an equilibrium state with $\left\langle\boldsymbol{\omega}_{\mathrm{f}}\right\rangle \sim\left(0,0, \omega_{\mathrm{eq}}\right)$ and $\left\langle\boldsymbol{L}_{\mathrm{f}}\right\rangle \sim \boldsymbol{L}_{\mathrm{eq}}\left(=I \boldsymbol{\omega}_{\mathrm{eq}}\right.$, where $\left.\omega_{\text {eq }}=\left(0,0, \omega_{\text {eq }}\right)\right)$ would be achieved after $N$ impacts of particles if the random component can be neglected, where $N$ satisfies the following relation:

$$
N\langle m\rangle\left(1-\varepsilon_{\mathrm{t}}\right) \sim \frac{\mathcal{K}+1}{C_{i}} M \sim M,
$$

where $C_{i} \sim 0.6-0.7$ for $i=x, y, z$ (Eqs. (35) and (40)) and we assume $\mathcal{K}=2 / 5$. The above $N$ can be regarded as the number of impacts required to erase the memory of the moonlet's initial rotation. On the other hand, the expectation value of $\boldsymbol{L}_{\mathrm{f}}^{2}$ after the above $N$ impacts can be written as

$$
\left\langle\boldsymbol{L}_{\mathrm{f}}^{2}\right\rangle=\left\langle\left(\boldsymbol{L}_{0}+\boldsymbol{L}\right)^{2}\right\rangle \sim \boldsymbol{L}_{\mathrm{eq}}^{2}+N\left\langle m^{2}\right\rangle\left\langle l^{2}\right\rangle,
$$

where we assumed $N \gg 1$ and used the relation $\left\langle\boldsymbol{L}^{2}\right\rangle \sim$ $\langle\boldsymbol{L}\rangle^{2}+N\left\langle m^{2}\right\rangle\left\langle l^{2}\right\rangle$ (Dones and Tremaine, 1993b; Ohtsuki and Ida, 1998). The first term in the last equation in Eq. (54) represents the systematic component that tends to lead the moonlet rotation toward the equilibrium state with $\left\langle\omega_{\mathrm{f}}\right\rangle \sim$ $\omega_{\text {eq }}$, while the second term accounts for the contribution of the random component. Using Eqs. (52) and (53), we can rewrite Eq. (54) as

$$
\left\langle\boldsymbol{L}_{\mathrm{f}}^{2}\right\rangle \sim \boldsymbol{L}_{\mathrm{eq}}^{2}\left\{1+S_{\mathrm{m}}^{2} S_{1}^{2}\right\}
$$

with

$$
S_{\mathrm{m}}^{2} \equiv \frac{\left\langle m^{2}\right\rangle}{M\langle m\rangle}
$$

and

$$
\begin{aligned}
S_{1}^{2} & \equiv \frac{\left\langle l^{2}\right\rangle}{\left(\mathcal{K} R^{2} \omega_{\mathrm{eq}}\right)^{2}} \cdot \frac{\mathcal{K} R^{2}\left(\omega_{\mathrm{eq}}-\omega_{0, z}\right)}{\left\langle l_{z}\right\rangle} \\
& =C_{1} \frac{\left(1-\varepsilon_{\mathrm{t}}\right) \sigma_{x}^{2}}{R^{2} \omega_{\mathrm{eq}}^{2}}
\end{aligned}
$$


where we used Eqs. (34) and (48), and $C_{1} \equiv C_{2}^{2} /\left\{C_{z}(\mathcal{K}+1)\right\}$. $C_{z}$ and $C_{2}$ are defined in Eqs. (35) and (49), respectively, and $C_{1}=1.122$ when $\beta=1 / 2$ and $\mathcal{K}=2 / 5$. Note that the final expression of $S_{1}^{2}$ does not depend on the moonlet's initial rotation rate $\left(\omega_{0, z}\right)$, as $\left\langle l_{z}\right\rangle \propto\left(\omega_{\mathrm{eq}}-\omega_{z}\right)$ (Eq. (34)). Equation (55) shows that the systematic component dominates the moonlet rotation when $S_{\mathrm{m}} S_{1}<1$, while the contribution of the random component can be significant if $S_{\mathrm{m}} S_{\mathrm{l}} \gtrsim 1$. Dones and Tremaine (1993b) obtained a relation similar to Eq. (55) for planetary rotation. In their formulation, the random component dominates planetary rotation when $S_{\mathrm{m}, \mathrm{DT}} S_{\mathrm{l}, \mathrm{DT}}>1$, where $S_{\mathrm{m}, \mathrm{DT}}^{2}=\left\langle m^{2}\right\rangle /(M\langle m\rangle)$ and $S_{1, \mathrm{DT}}^{2}=\left\langle l^{2}\right\rangle /\left\langle l_{z}\right\rangle^{2}$. In these expressions defined by Dones and Tremaine (1993b), $\langle m\rangle$ and $\left\langle m^{2}\right\rangle$ are the mean and mean square masses of accreted planetesimals, and $M$ in their case is the mass of the planet and is given by $M=N\langle m\rangle$. On the other hand, $M$ in Eq. (56) is independent of the mass distribution of impacting particles and is assumed to be kept constant in the present work. Also, $S_{1, \mathrm{DT}}^{2}$ is expressed only in terms of the angular momentum distribution of accreted planetesimals, while $S_{1}^{2}$ in Eq. (57) depends also on the size and rotation rate of the moonlet (Note that $\mathcal{K} R^{2} \omega$ represents the moonlet's spin angular momentum per unit mass).

If all the impacting particles have the same mass $m$, we have

$$
S_{\mathrm{m}}=(m / M)^{1 / 2}
$$

In a more realistic case where impacting particles have a size distribution given by $N(R) d R \propto R^{-q} d R$ with $q \sim 3$ for $m_{0} \leq m \leq m_{1}$ ( $m_{0}$ and $m_{1}$ are the mass of the smallest and the largest particles), we find

$$
S_{\mathrm{m}} \sim 0.5 \times\left(\frac{m_{1}}{M}\right)^{1 / 2} .
$$

On the other hand, Eq. (57) shows that the relative importance of the systematic and the random components also depends on the values of $\varepsilon_{\mathrm{t}}$ and the magnitude of particles' velocity dispersion relative to the moonlet's equilibrium rotation velocity $R \omega_{\mathrm{eq}}$, which is on the order of the Kepler shear across the moonlet's radius $(\sim R \Omega)$, as $\mathcal{O}\left(\omega_{\mathrm{eq}}\right) \sim \Omega$ (Eq. (35)).

In the above, we assumed that the mass of an impacting particle is much smaller than the moonlet's mass, and we replaced $\mu$ and $R_{1}$ in Eq. (14) with $m_{2}$ and $R_{\mathrm{p}}\left(=R_{1}+R_{2}\right)$, respectively. When the particle mass $\left(m_{i}\right)$ is not negligible compared to the moonlet's mass $(M)$, each term of the r.h.s. of Eq. (50) needs to be multiplied by a factor

$$
f\left(\mu_{i}^{\prime}\right) \equiv\left(1+\mu_{i}^{\prime}\right)^{-1}\left(1+\mu_{i}^{\prime 1 / 3}\right)^{-1}
$$

where $\mu_{i}^{\prime}=m_{i} / M$.

In planetary rings with low optical depth, particles' velocity dispersion can be approximated by $\sigma_{x} \sim \max \left\{v_{\mathrm{e}},(2-\right.$ 3) $R_{2} \Omega$ \} in the gravitating case and $\sigma_{x} \sim(2-3) R_{2} \Omega$ in the non-gravitating case $\left(v_{\mathrm{e}}\right.$ and $R_{2}$ are the escape velocity and the radius of a particle), if the moonlet is small enough to neglect its perturbation on particles' velocities (e.g., Salo, 1995; Ohtsuki, 1999). Using Eq. (35), we then find that $S_{1} \sim(2-5)(m / M)^{1 / 3}$ in non-gravitating rings of equalsized particles with $\varepsilon_{\mathrm{t}} \sim 0.6-0.9$ and $m \ll M$. Thus, taking into account the correction factor $f\left(\mu^{\prime}\right)\left(\mu^{\prime}=m / M\right)$, we obtain

$$
S_{\mathrm{m}} S_{1} \sim(2-5) \times\left(f\left(\mu^{\prime}\right)\right)^{1 / 2}\left(\frac{m}{M}\right)^{5 / 6} .
$$

Figure 2 shows the plots of $S_{\mathrm{m}} S_{1}$ in the non-gravitating, equal-sized-particle case with $\varepsilon_{\mathrm{t}}=0.7$ and $\sigma_{x}=2 R_{2} \Omega$, as a function of $m / M$. The above estimate shows that $S_{\mathrm{m}} S_{1}<1$ and the systematic component dominates the moonlet rotation as long as the mass of the largest impactors is much smaller than the moonlet's mass. In this case, $\left\langle\omega_{\mathrm{f}}\right\rangle \sim \boldsymbol{\omega}_{\mathrm{eq}}$, and a slow prograde rotation of the moonlet can be expected. On the other hand, the random component can be significant, if the mass of impacting particles is comparable to the moonlet's mass and/or the velocity dispersion of particles is much larger than the Kepler shear across the moonlet's radius (e.g., by external perturbation). In this case, we find

$$
\frac{\left\langle\omega_{\mathrm{f}}^{2}\right\rangle^{1 / 2}}{\Omega} \sim S_{\mathrm{m}} S_{1}^{\prime},
$$

where $S_{1}^{\prime 2} \equiv C_{1}\left(1-\varepsilon_{\mathrm{t}}\right) \sigma_{x}^{2} /\left(R^{2} \Omega^{2}\right)=\left(\omega_{\mathrm{eq}}^{2} / \Omega^{2}\right) S_{1}^{2}$. Equation (62) shows that the moonlet can have both prograde and retrograde rotations, and that the rotation rate would depend on the values of the mass of the largest impactors relative to the moonlet's mass $\left(S_{\mathrm{m}}\right)$ and the velocity dispersion of the impactors relative to the Kepler shear across the moonlet's $\operatorname{radius}\left(S_{1}^{\prime}\right)$.

\section{Comparison with $\mathbf{N}$-body Simulation}

In order to confirm the validity of the formulation for moonlet rotation derived in Section 4 and the analytic results described in Section 3, we performed a local N-body simulation for a system of a moonlet embedded in planetary rings, neglecting their mutual gravity. The numerical method we

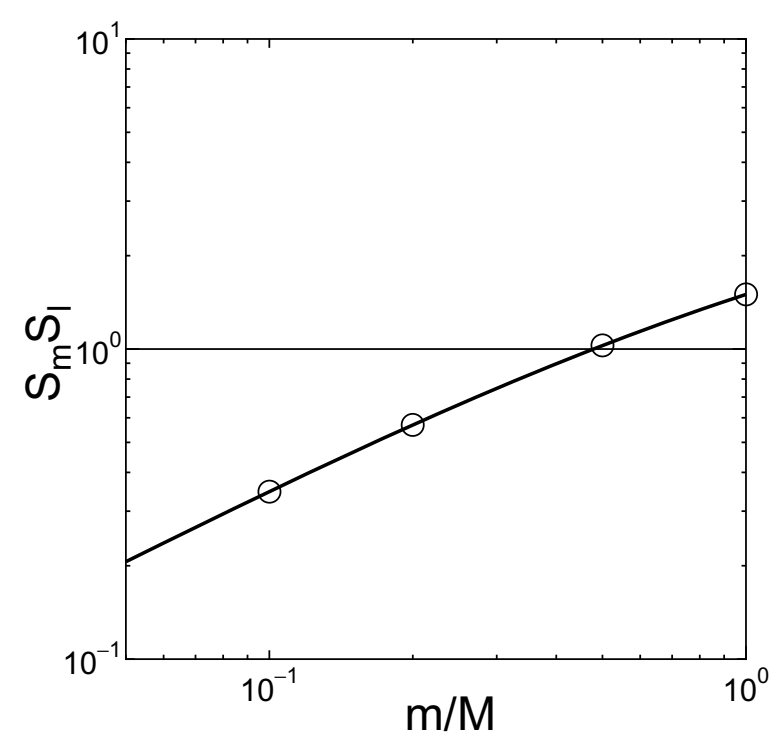

Fig. 2. The values of $S_{\mathrm{m}} S_{\mathrm{l}}$ defined by Eqs. (56) and (57) in non-gravitating rings of equal-sized particles with $\varepsilon_{\mathrm{t}}=0.7$ are shown as a function of $m / M$, where $m$ and $M$ are the mass of a particle and the moonlet, respectively. The open circles show the values of $m / M$ for which N-body simulation was performed in Fig. 3 (see Section 5). 

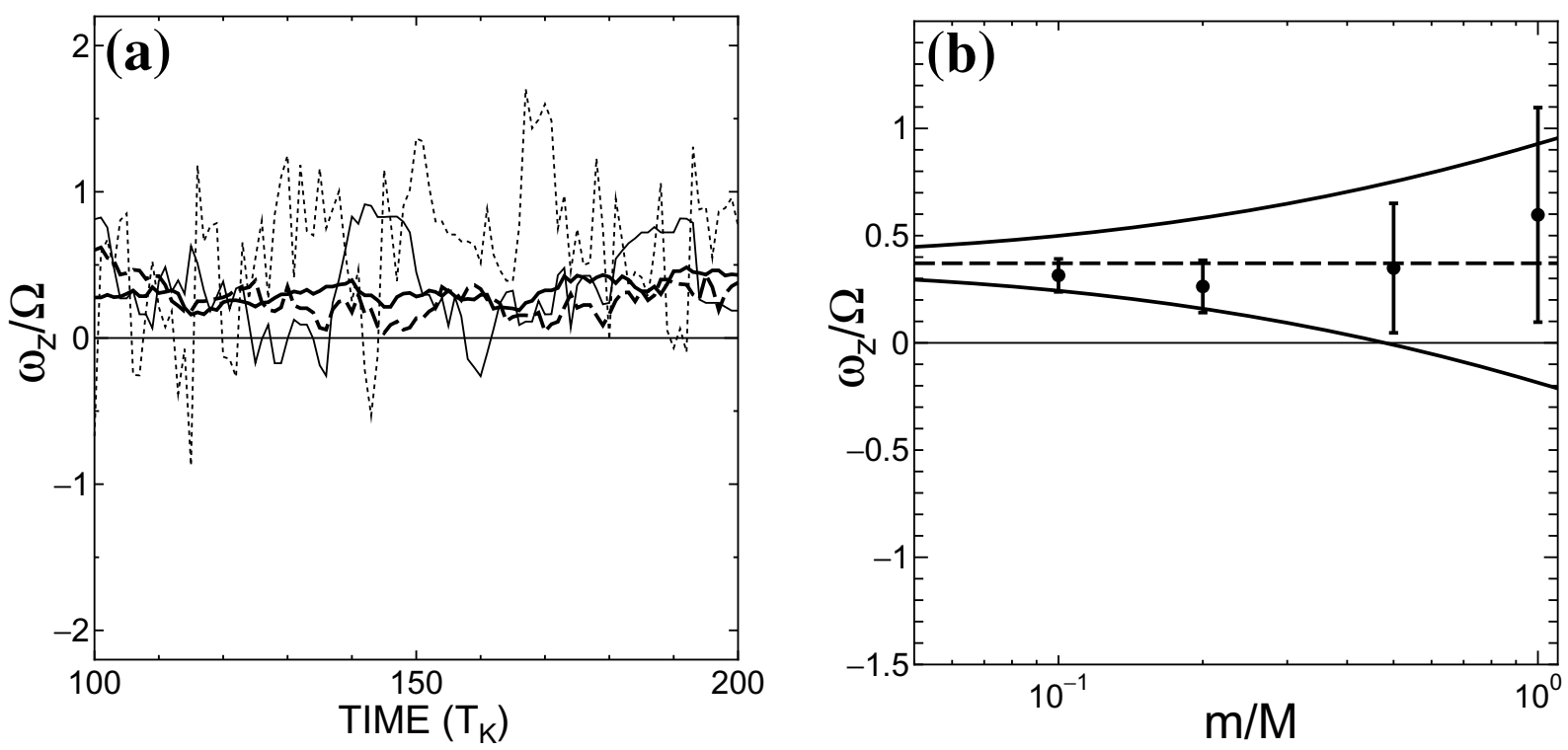

Fig. 3. (a) Numerical results of N-body simulation for the evolution of the $z$-component of the rotation rate (scaled by the Keplerian orbital frequency $\Omega$ ) of a non-gravitating moonlet embedded in planetary rings of equal-sized particles. Four cases with different particle-moonlet mass ratios are shown; $m / M=0.1$ (thick solid line), 0.2 (thick dashed line), 0.5 (thin solid line), and 1 (thin dotted line). (b) Mean value and dispersions of the $z$-component of the moonlet's rotation rate. The thin dashed and the thin solid lines represent the equilibrium rotation rate and the dispersion obtained by the analytic calculations, while the solid circles and the error bars show the mean values and the dispersions obtained from the results of N-body simulation.

used is similar to our previous one described in Ohtsuki and Emori (2000).

Figure 3(a) shows the evolution of the $z$-component of the moonlet's rotation rate ( $T_{\mathrm{K}}$ is the orbital period). In this simulation, one moonlet is embedded in a swarm of one thousand ring particles with their optical depth of 0.1 , and their restitution coefficients in normal and tangential directions are 0.5 and 0.7 , respectively. Four lines show the results with different particle-moonlet mass ratios $(\mathrm{m} / \mathrm{M}=$ $0.1,0.2,0.5$, and 1$)$. We show the plots for $100 T_{\mathrm{K}} \leq t \leq$ $200 T_{\mathrm{K}}$ to demonstrate the quasi-equilibrium behavior of the system. When the mass ratio is small $(0.1-0.2)$, we find that the contribution of the random component is negligible or relatively small, and the values of $\omega_{z}$ show only slight fluctuation around its equilibrium value of $\sim(0.3-0.4) \times$ $\Omega$. On the other hand, when the mass of the particle is comparable to that of the moonlet (i.e., $m / M \gtrsim 0.5$ ), the random component becomes significant and the rotation rate shows large fluctuation. In this case, it takes on even negative values (i.e., retrograde rotation). These results are consistent with the above analytic prediction (Fig. 2) based on our new formulation.

In order to further compare the analytic and the N-body results quantitatively, we calculated the dispersion of the moonlet's rotation rate in both cases. In the case of the analytic calculations, the dispersion is given by Eq. (62). On the other hand, in the case of the N-body simulation, we calculated the mean values and the dispersions directly from the numerical results shown in Fig. 3(a). Figure 3(b) shows the plots of the dispersions around the mean value as a function of $m / M$. The horizontal dashed line and the two solid lines represent the mean value and the dispersion calculated by the analytic results. The solid circles and the vertical lines show the mean values and the dispersions obtained by $\mathrm{N}$ body simulation. These results show quite good agreement between the analytic and the N-body results.

\section{Conclusions and Discussion}

In the present work, we have evaluated analytically the mean and mean square spin angular momenta that a moonlet acquires by inelastic collisions of ring particles, taking account of the Rayleigh distribution of particles' eccentricities and inclinations. Our results for the three components of the mean angular momentum presented in Section 3 suggest an equilibrium rotation of a moonlet in the prograde direction that is slower than the synchronous rotation. However, in addition to the above systematic component arising from an average of the angular momentum provided by a number of small impacts, the random component that comes from large impacts also needs to be taken into account. We have derived a new formulation that allows a unified treatment of the systematic and the random components of moonlet rotation. In the non-gravitating case, we have found that the contribution of the random component would be significant when the mass of largest impactors is comparable to the moonlet's mass and/or the velocity dispersion of particles is larger than the Kepler shear across the moonlet's radius. We also confirmed quantitative agreement between the analytic results and those obtained by N-body simulation. On the other hand, the assumption that a moonlet is on a circular orbit is not a good approximation when the mass of an impacting particle is comparable to the moonlet's mass. We also have to consider energy exchange between rotation and random motion (Salo, 1987b). All these effects become important for rotation of ring particles with a continuous size distribution. The observation of thermal emission from Saturn's rings by the Voyager spacecraft suggests slow rotation of ring particles (Hanel et al., 1981). Since it is expected that the composite infrared spectrometer on the Cassini spacecraft will obtain more detailed information about rotation states of Saturn's 
ring particles (e.g., Spilker et al., 2002), further theoretical studies on rotation of ring particles and moonlets are needed for direct comparison with these observations.

In the present work, we focused on the non-gravitating case, and neglected the effect of mutual gravity between a moonlet and ring particles. Such an approximation is valid if their relative velocity is at least a few times larger than the escape velocity of the moonlet. However, in general cases, mutual gravity between ring particles plays an important role in their velocity distribution and structure formation, especially at the outer parts of planetary rings where the tidal effect becomes less important and in the case of large optical depths where collective effects become dominant (e.g., Salo, 1995; Ohtsuki, 1993, 1999; Daisaka and Ida, 1999; Ohtsuki and Emori, 2000). The effect of gravity also affects the moonlet rotation by particle collisions, as it significantly enhances the number of collisions (Morishima and Salo, 2004; Ohtsuki, 2004). On the basis of the formulation derived in the present paper, we examine the rotation of a gravitating moonlet due to particle collisions in a separate paper (Ohtsuki, 2004), where we present numerical results of three-body orbital integration that takes into account the effects of gravity and velocity distribution of ring particles. The results of the present paper may also be applied to asteroid rotation, where the effect of mutual gravity is less important owing to high relative velocities, although catastrophic fragmentation needs to be taken into account in this case.

Acknowledgments. I thank Sei-ichiro Watanabe for his prompt and thoughtful review, which greatly improved the manuscript. Comments by Ryuji Morishima were very helpful in the significant improvement of the formulation and presentation in Sections 4 and 5. I also thank Larry Esposito, Jack Lissauer, Sho Sasaki, and Glen Stewart for discussion and comments. This work was supported by the Cassini Project and NASA's Planetary Geology and Geophysics Program. Part of numerical calculations was performed at the Center for Planning and Information Systems of Japan Aerospace Exploration Agency.

\section{Appendix}

In this appendix, we describe the derivation of approximate analytic expressions of the integrals in Eqs. (9), (33), and (47). Greenzweig and Lissauer (1992) derived an approximate analytic expression of the integral in Eq. (9). The idea behind their method of derivation is that the elliptic integral is a slowly varying function of its argument in the integration range, therefore it can be taken out of the integral and its argument can be replaced by an average value. In the first-order approximation, $I(\beta)$ given in Eq. (9) can be written as

$$
\begin{aligned}
I(\beta) & =4 \int E(A(\chi)) W_{1}(\chi) d \chi \\
& \simeq 4 E\left(\overline{A_{1}}\right) \int W_{1}(\chi) d \chi
\end{aligned}
$$

where

$$
\begin{aligned}
& A(\chi) \equiv \frac{\sqrt{3}}{2} \sqrt{1-\chi^{2}} \\
& W_{1}(\chi) \equiv \frac{1}{\left\{\beta+\left(\beta^{-1}-\beta\right) \chi^{2}\right\}^{2}},
\end{aligned}
$$

and

$$
\overline{A_{1}} \equiv \frac{\int A(\chi) W_{1}(\chi) d \chi}{\int W_{1}(\chi) d \chi} .
$$

The integrals in Eq. (A.3) can be evaluated analytically. When $\beta<1$, we have

$$
\begin{aligned}
& \int A(\chi) W_{1}(\chi) d \chi=\frac{\sqrt{3} \pi}{8 \beta} \\
& \mathcal{I}_{w 1} \equiv \int W_{1}(\chi) d \chi=\frac{1}{2}(1+T(\beta))
\end{aligned}
$$

where

$$
T(\beta) \equiv \frac{\tan ^{-1} \sqrt{\beta^{-2}-1}}{\beta \sqrt{1-\beta^{2}}} .
$$

Thus, we have (Greenzweig and Lissauer, 1992)

$$
\overline{A_{1}}=\frac{\sqrt{3} \pi}{4 \beta}(1+T(\beta))^{-1} .
$$

When $\beta=1$, we have $\overline{A_{1}}=\sqrt{3} \pi / 8$ and $\mathcal{I}_{w 1}=1$. Greenzweig and Lissauer (1992) also calculated the second-order approximation for $I(\beta)$. Here, we only show the calculations for the first-order approximation, because they provide sufficiently accurate results, as shown below. From Eqs. (A.1), (A.4), and (A.6), we obtain (Greenzweig and Lissauer, 1992)

$$
I(\beta) \simeq 4 E\left(\overline{A_{1}}\right) \mathcal{I}_{w 1} \text {. }
$$

The asymptotic form of $I(\beta)$ in the limit of $\beta \ll 1$ can be easily obtained from Eqs. (A.4), (A.5), and (A.6) as

$$
I(\beta) \simeq \pi E(\sqrt{3} / 2) / \beta .
$$

Approximate analytic expressions for other integrals in Eqs. (33), (38), and (47) can be also obtained in similar manners. Those for $I_{t z}(\beta), I_{x^{2}+y^{2}}(\beta), I_{y^{2}+z^{2}}(\beta)$, and $I_{z^{2}+x^{2}}(\beta)$ are

$$
\begin{aligned}
& I_{t z}(\beta) \simeq\left(2 E\left(\overline{A_{1}}\right)-\frac{1}{2} K\left(\overline{A_{1}}\right)+\frac{1}{6 \pi}\right) \mathcal{I}_{w 1}-K\left(\overline{A_{1}}\right) \mathcal{I}_{2 w 1} \\
& I_{x^{2}+y^{2}}(\beta) \simeq 3 E\left(\overline{A_{1}}\right) \mathcal{I}_{w 1}-K\left(\overline{A_{1}}\right) \mathcal{I}_{2 w 1} \\
& I_{y^{2}+z^{2}}(\beta) \simeq\left(\frac{5}{3} E\left(\overline{A_{1}}\right)+\frac{1}{3} K\left(\overline{A_{1}}\right)\right) \mathcal{I}_{w 1}+K\left(\overline{A_{1}}\right) \mathcal{I}_{2 w 1} \\
& I_{z^{2}+x^{2}}(\beta) \simeq\left(\frac{10}{3} E\left(\overline{A_{1}}\right)-\frac{1}{3} K\left(\overline{A_{1}}\right)\right) \mathcal{I}_{w 1}
\end{aligned}
$$

where

$$
\begin{aligned}
\mathcal{I}_{2 w 1} \equiv \int \chi^{2} W_{1}(\chi) d \chi & \begin{cases}\frac{T(\beta)-1}{2\left(\beta^{-2}-1\right)} & \text { for } \beta<1 \\
& = \begin{cases}\frac{1}{3} & \text { for } \beta=1\end{cases} \end{cases}
\end{aligned}
$$


In order to derive the approximate exprresions for $I_{2}(\beta)$ and $I_{z 2}(\beta)$, we introduce

$$
W_{2}(\chi) \equiv \frac{\beta}{\left\{\beta+\left(\beta^{-1}-\beta\right) \chi^{2}\right\}^{3}},
$$

and define $\mathcal{I}_{w 2}, \mathcal{I}_{2 w 2}$, and $\overline{A_{2}}$, replacing $W_{1}$ with $W_{2}$ in the definitions of $\mathcal{I}_{w 1}, \mathcal{I}_{2 w 1}$, and $\overline{A_{1}} \cdot \mathcal{I}_{w 2}, \mathcal{I}_{2 w 2}$, and $\overline{A_{2}}$ for $\beta<1$ are evaluated as

$$
\begin{aligned}
& \mathcal{I}_{w 2}=\frac{1}{8}\left(3+2 \beta^{2}+3 T(\beta)\right), \\
& \mathcal{I}_{2 w 2}=\frac{\beta^{2}}{8\left(1-\beta^{2}\right)}\left(1-2 \beta^{2}+T(\beta)\right), \\
& \overline{A_{2}}=\frac{\sqrt{3} \pi\left(3+\beta^{2}\right)}{4 \beta\left(3+2 \beta^{2}+3 T(\beta)\right)} .
\end{aligned}
$$

For $\beta=1$, we have $\mathcal{I}_{w 2}=1, \mathcal{I}_{2 w 2}=1 / 3$, and $\overline{A_{2}}=\sqrt{3} \pi / 8$. Using these, we obtain

$$
\begin{aligned}
& I_{2}(\beta) \simeq\left(4 E\left(\overline{A_{2}}\right)-3 F\left(\overline{A_{2}}\right)\right) \mathcal{I}_{w 2}+3 F\left(\overline{A_{2}}\right) \mathcal{I}_{2 w 2} \\
& I_{z 2}(\beta) \simeq\left(2 E\left(\overline{A_{2}}\right)-\frac{3}{2} F\left(\overline{A_{2}}\right)\right)\left(\mathcal{I}_{w 2}-\mathcal{I}_{2 w 2}\right) .
\end{aligned}
$$

Note that $\overline{A_{1}}$ and $\overline{A_{2}}$ as well as their elliptic integrals such as $E\left(\overline{A_{1}}\right)$ and $E\left(\overline{A_{2}}\right)$ agree with each other within $4 \%$ for $\beta \leq 1$, thus $\overline{A_{1}}$, which has a simpler expression, may be used in Eqs. (A.13) instead of $\overline{A_{2}}$.

In Fig. 1, we plot the values of the integrals calculated by these approximate analytic expressions (Eqs. (A.9) and (A.13)) by the open circles. We confirmed that they agree with those obtained by direct numerical integration within $5 \%$ for the entire range of $\beta$ shown in Fig. 1.

In the limit of $\beta \ll 1$, the above analytic expressions have the following asymptotic forms:

$$
\begin{aligned}
& I_{t z}(\beta) \simeq\left(2 E(\sqrt{3} / 2)-\frac{1}{2} K(\sqrt{3} / 2)+\frac{1}{6 \pi}\right) \frac{\pi}{4 \beta}, \\
& I_{x^{2}+y^{2}}(\beta) \simeq 3 E(\sqrt{3} / 2) \frac{\pi}{4 \beta}, \\
& I_{y^{2}+z^{2}}(\beta) \simeq(5 E(\sqrt{3} / 2)+K(\sqrt{3} / 2)) \frac{\pi}{12 \beta}, \\
& I_{z^{2}+x^{2}}(\beta) \simeq(10 E(\sqrt{3} / 2)-K(\sqrt{3} / 2)) \frac{\pi}{12 \beta}, \\
& I_{2}(\beta) \simeq 2 I_{z 2}(\beta) \simeq\left(E(\sqrt{3} / 2)-\frac{3}{4} F(\sqrt{3} / 2)\right) \frac{3 \pi}{4 \beta} .
\end{aligned}
$$

\section{References}

Araki, S., The dynamics of particle disks. II. Effects of spin degrees of freedom, Icarus, 76, 182-198, 1988.

Araki, S., The dynamics of particle disks. III. Dense and spinning particle disks, Icarus, 90, 139-171, 1991.

Araki, S. and S. Tremaine, The dynamics of dense particle disks, Icarus, $\mathbf{6 5}$, 83-109, 1986.
Daisaka, H. and S. Ida, Spatial structure and coherent motion in dense planetary rings induced by self-gravitational instability, Earth Planets Space, 51, 1195-1213, 1999.

Dones, L. and S. Tremaine, Why does the Earth spin forward?, Science, $\mathbf{2 5 9}$ 350-354, 1993a.

Dones, L. and S. Tremaine, On the origin of planetary spins, Icarus, 103, 67-92, 1993b.

Giuli, R. T., On the rotation of the Earth produced by gravitational accretion of particles, Icarus, 8, 301-323, 1968.

Greenzweig, Y. and J. J. Lissauer, Accretion rates of protoplanets, Icarus, 87, 40-77, 1990.

Greenzweig, Y. and J. J. Lissauer, Accretion rates of protoplanets. II. Gaussian distribution of planetesimal velocities, Icarus, 100, 440-463, 1992.

Hanel, R., B. Conrath, F. M. Flasar, V. Kunde, W. Maguire, J. Pearl, J. Pirraglia, R. Samuelson, L. Herath, M. Allison, D. Cruikshank, D. Gautier, P. Gierasch, L. Horn, R. Koppany, and C. Ponnamperuma, Infrared observations of the Saturnian System from Voyager 1, Science, 212, 192-200, 1981.

Ida, S. and K. Nakazawa, Did rotation of the protoplanets originate from the successive collisions of planetesimals?, Icarus, 86, 561-573, 1990.

Lissauer, J. J. and D. M. Kary, The origin of the systematic component of planetary rotation. I. Planet on a circular orbit, Icarus, 94, 126-159, 1991 .

Lissauer, J. J. and V. S. Safronov, The random component of planetary rotation, Icarus, 93, 288-297, 1991.

Lissauer, J. J., A. F. Berman, Y. Greenzweig, and D. M. Kary, Accretion of mass and spin angular momentum by a planet on an eccentric orbit, Icarus, 127, 65-92, 1997.

Lissauer, J. J., L. Dones, and K. Ohtsuki, Origin and evolution of terrestrial planet rotation, in Origin of the Earth and Moon, edited by R. M. Canup and K. Righter, pp. 101-112, University of Arizona Press, Tucson, 2000.

Morishima, R. and H. Salo, Spin rates of small moonlets embedded in planetary rings: I. Three-body calculations, Icarus, 167, 330-346, 2004.

Nakazawa, K., S. Ida, and Y. Nakagawa, Collisional probability of planetesimals revolving in the solar gravitational field. I. Basic formulation, Astron. Astrophys., 220, 293-300, 1989.

Ohtsuki, K., Equilibrium velocities in planetary rings with low optical depth, Icarus, 95, 265-282, 1992

Ohtsuki, K., Capture probability of colliding planetesimals: Dynamical constraints on accretion of planets, satellites, and ring particles, Icarus, $\mathbf{1 0 6}$ 228-246, 1993.

Ohtsuki, K., Evolution of particle velocity dispersion in a circumplanetary disk due to inelastic collisions and gravitational interactions, Icarus, 137, 152-177, 1999.

Ohtsuki, K., On the rotation of a moonlet embedded in planetary rings, Icarus, 2004 (in press).

Ohtsuki, K. and H. Emori, Local N-body simulations for the distribution and evolution of particle velocities in planetary rings, Astron. J., 119 403-416, 2000.

Ohtsuki, K. and S. Ida, Planetary rotation by accretion of planetesimals with nonuniform spatial distribution formed by the planet's gravitational perturbation, Icarus, 131, 393-420, 1998.

Salo, H., Collisional evolution of rotating, non-identical particles, Moon Planets, 38, 149-181, 1987a.

Salo, H., Numerical simulations of collisions between rotating particles, Icarus, 70, 37-51, 1987b.

Salo, H., Simulations of dense planetary rings. III. Self-gravitating identical particles, Icarus, 117, 287-312, 1995.

Spilker, L., C. Ferrari, M. R. Showalter, J. N. Cuzzi, R. Achterberg, J. Pearl, M. Flasar, V. Kunde, S. Edberg, B. Wallis, J. Aiello, S. Edgington, and Cassini CIRS Investigation Team, Cassini CIRS observations of Saturn's rings, Bull. Amer. Astron. Soc., 34, 900, 2002.

Tanikawa, K., S. Manabe, and R. Broucke, On the origin of the spin angular momentum by accretion of planetesimals: Property of collision orbits, Icarus, 79, 208-222, 1989.

Weidenschilling, S. J., C. R. Chapman, D. R. Davis, and R. Greenberg, Ring particles: Collisional interactions and physical nature, in Planetary Rings, edited by R. Greenberg and A. Brahic, pp. 367-415, University of Arizona Press, Tucson, 1984

K. Ohtsuki (e-mail: ohtsuki@lasp.colorado.edu) 